\title{
MULTIPLE MUTATION IN CHLAMYDOMONAS REINHARDI
}

\author{
J. N. HARTSHORNE \\ Department of Botony, University of Manchester
}

\section{INTRODUCTION}

Received 10.xi.54

THE rate of natural or induced mutation at most loci is so low that it is uncommon to find an individual in which simultaneous mutation at more than one locus has occurred. This paper will describe the genetical analysis of a mutant clone of Chlamydomonas reinhardi which seems to have arisen by simultaneous mutation at three loci.

\section{MATERIALS AND METHODS}

C. reinhardi is a heterothallic, unicellular green alga. Genetical evidence indicates that the vegetative cells are haploid. When two clones of opposite mating-type are mixed together under suitable conditions, sexual fusion occurs and zygotes are formed. The zygote represents the only diploid stage in the life-cycle, and meiosis occurs when the zygote germinates. The strains used in this work were isolated from soil by Professor G. M. Smith of Stanford University, California, who kindly supplied the author with cultures.

In genetical experiments with $C$. reinhardi the techniques used have been modified from those of Smith and Regnery (1950), and will be described in detail elsewhere. The life-cycle can be completed within three weeks. Zygotes germinate readily and the products of germination, usually four or eight cells, can be isolated. The tetrad is not ordered. The majority of zygotes germinated produce eight cells, but since these represent four pairs all zygotes can be treated as though they produce four cells.

\section{THE EYELESS TYPE}

Cultures of wild-type cells in liquid medium were irradiated with ultra-violet light in order to produce mutations, after which the cells were spread over the surface of nutrient agar plates. The plates were kept in continuous light and after several days colonies, each produced from a single irradiated cell, were visible on the agar surface. Cells from these colonies were examined under the microscope for morphological differences from wild-type, and in one clone it was found that the characteristic red eyespot was lacking. This character has remained constant through repeated sub-cultures and is given the name eyeless (symbol ey).

The original eyeless clone was of minus mating-type, and it was crossed with the wild-type plus strain. The products of $5^{8}$ zygotes were examined for presence of the eyespot and in all cases there was $2: 2$ segregation for presence and absence. It was therefore concluded that absence of the eyespot was due to a single gene difference.

From the progeny of this first cross, an eyeless clone of plus matingtype was selected and crossed with wild-type minus. The products of 36 zygotes were examined and again showed regular $2: 2$ segregation for presence and absence of the eyespot. 


\section{THE SMALL-COLONY TYPE}

A striking feature of progenies from both these crosses was the appearance of certain colonies which were smaller and paler than normal. The difference in size and colour was quite unmistakable. Normal colonies were about $2 \mathrm{~mm}$. in diameter after a week's growth and were a rich dark green colour ; small colonies, however, rarely exceeded $0.5 \mathrm{~mm}$. in diameter and were pale yellow. Each set of four colonies from a single zygote included either two, one or none which were of this type. In table I the zygote progenies from the two crosses described above have been classified according to the ratio of normal to small colonies.

TABLE I

Results of crosses between eyeless (ey) and wild-type $\left(\mathrm{ey}^{+}\right)$

\begin{tabular}{|c|c|c|c|c|c|c|c|}
\hline \multirow{2}{*}{\multicolumn{3}{|c|}{$\begin{array}{c}\text { Number and description } \\
\text { of cross }\end{array}$}} & & \multirow{2}{*}{$\begin{array}{l}\text { Number of } \\
\text { zygotes } \\
\text { analysed }\end{array}$} & \multicolumn{3}{|c|}{$\begin{array}{c}\text { Number of zygotes producing } \\
\text { normal and small colonies } \\
\text { in the ratio }\end{array}$} \\
\hline & & & & & $4: 0$ & $3: 1$ & $2: 2$ \\
\hline \multirow{3}{*}{$\begin{array}{r}\text { 1. } y-\times e y^{+}+ \\
\text {2. } e y+\times e y^{+}- \\
\text {Totals }\end{array}$} & & . & & $5^{8}$ & 2 & 54 & 2 \\
\hline & & . & • & $3^{6}$ & 2 & 32 & 2 \\
\hline & $\cdot$ & . & • & 94 & 4 & 86 & 4 \\
\hline
\end{tabular}

As was mentioned above, all zygote progenies were examined for presence or absence of the eyespot. Not only did each progeny show $2: 2$ segregation for this character, but the small colonies invariably consisted of cells lacking eyespots. It should be noted, however, that colonies of cells without eyespots were not necessarily small.

These observations led to a hypothesis which was then tested by further crosses. The hypothesis is that a gene small-colony (symbol sc) is responsible for reduced colony size, and that this gene is suppressed by the wild-type allele at the eyeless locus $\left(e y^{+}\right)$but not by the mutant allele $(e y)$. If this is so, the parental genotypes in cross no. I (table I) may be amplified to read as follows :

$$
\text { ey } s c^{+}-\times e y+s c+
$$

Zygotes from this cross would yield progenies of three types (ignoring for the moment the inheritance of mating-type) :

I. Segregation into the two parental genotypes;

$$
\begin{aligned}
& e y+s c \text { normal colony } \\
& e y+s c \quad, \quad, \\
& \text { ey } \mathrm{sc}^{+}, \text {, , } \\
& \text { ey } \mathrm{sc}^{+}, \text {, , }
\end{aligned}
$$


2. Segregation into four genotypes;

$$
\begin{aligned}
& e y+s c+\text { normal colony } \\
& e y+s c, ", \\
& e y \quad s c+, ", \\
& e y \text { sc small colony }
\end{aligned}
$$

3. Segregation into two non-parental genotypes ;

$$
\begin{aligned}
& e y+s c+\text { normal colony } \\
& e y+s c+, ", \\
& e y \text { sc small colony } \\
& e y \text { sc },, "
\end{aligned}
$$

These three types of segregation would account for the three types of progeny obtained from crosses I and 2 (table 1 ).

Experiments designed to test the hypothesis were carried out using sets of clones from the zygote progenies of cross no. I. The first set, from zygote $\mathrm{O}_{4}$, consisted of the following eight clones :
(a) and (g)
$e y^{+}+$normal colony
(b) and (h)
ey+- , , ,
(c) and (d)
ey - , , ,
(e) and (f)
ey,$+ \quad$,

According to the hypothesis this set belongs to type I (see above), and all clones carrying the $e^{+}$gene should also carry sc, though this is not expressed. If each clone with eyespots were crossed with an eyeless strain there should be produced in each case some recombinant clones with small colonies. Crosses of this sort were made, with the results shown in table 2 (crosses $3-6$ ).

Zygote $S_{2}$ gave rise to the following eight clones :
(a) and (c)
(b) and (g)
(d), (e), (f)
ey $(-)^{*}$ small colony
ey - normal colony
(h) $e y^{+}+$
",

This set of clones shows a $3:$ I ratio of normal and small colonies, and so belongs to type 2 . Clones (d), (e), (f) and (h) are phenotypically all alike, but according to hypothesis two of them should carry $s c$ and two $s c^{+}$. By crossing them with an eyeless strain the genotypic difference should become apparent; recombinant clones with small colonies should be produced from only two of the crosses. All four crosses were made and the results are shown in table 2 (crosses 7-10).

Zygote $\mathrm{V}_{4}$ gave rise to the following eight clones :
(a) and (h)
ey (-)
small colony
(b) and (c)
$e y^{+}-$normal colony
(d) and (e)
ey +
(f) and (g)
$e y^{+}+$

,",

This set also belongs to type 2, and of the four clones with eyespots

* Brackets around the mating-type symbol indicate that in this case mating-type was determined by inference. In only a few cases has it been possible to determine directly the mating-type of small-colony clones. 
only two are expected, by hypothesis, to carry sc. In this case there is the further expectation that the two carrying $s c$ are either (b) and (c) or (f) and (g) since mating-type tests have shown that these are sister clones. Results of the crosses made with these clones are shown in table 2 (crosses II-I4).

Zygote EEI gave rise to the following eight clones :
(a) and (b)
(c) and (d)
$e y^{+}-$normal colony
(e), (f), (g) and
$e y^{+}+$
(h) ey
small colony

This set belongs to type 3. All eyeless clones have small colonies, and it is therefore to be expected that none of the clones with eyespots

TABLE 2

Results of crosses made to study the inheritance of the small-colony type

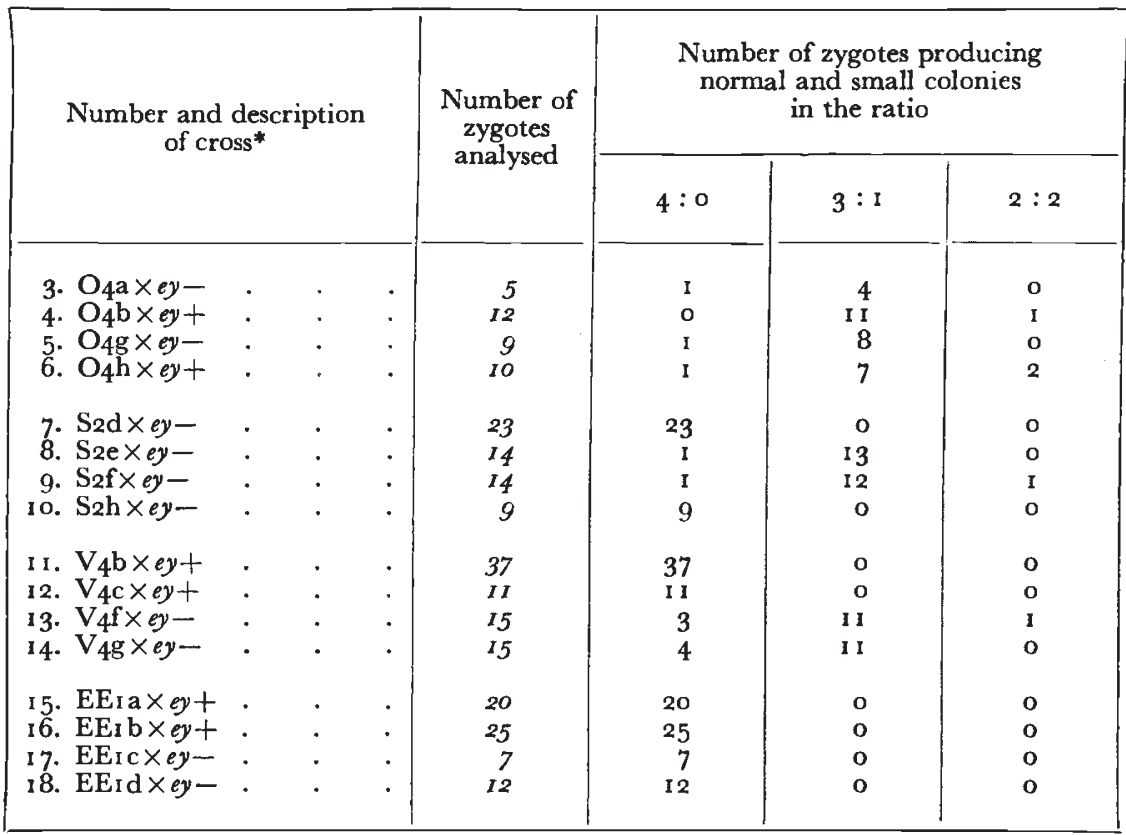

* The first parent in cross no. 3 was clone (a) from zygote $\mathrm{O}_{4}$; this has been written as $\mathrm{O}_{4}$ a. Similar abbreviation has been used in describing other parent strains.

carry sc. If this were so none of the clones with eyespots should produce small-colony clones when crossed with an eyeless strain. The results of such crosses are shown in table 2 (crosses $15^{-18}$ ).

Table 2 shows that all crosses yielded results in agreement with the hypothesis, and hence there is evidence for the existence of the $s c$ gene and for its behaviour in the manner postulated.

The ey and sc genes do not appear to be linked. Table I shows that parental and non-parental two-type segregations are equally frequent in crosses segregation for alleles at both loci, ar this is as expected when dealing with two unlinked loci. 
The frequency of four-type segregations (9I 49 per cent.) significantly exceeds 66.67 per cent. This indicates that there is more than 50 per cent. crossing-over between one of the loci and its centromere resulting in this locus showing more than 66.67 per cent. seconddivision segregation (Whitehouse, 1949). Since there is evidence that the ey locus is close to its centromere (see p. 245) it is probably the $s c$ locus which has this high frequency of second-division segregation.

\section{THE SLOW-GROWTH TYPE}

Table 2 described the progenies from certain zygotes as consisting of normal colonies only. This means that all the colonies were dark green in colour and capable of reaching a diameter of at least $2 \mathrm{~mm}$. ; there is no possibility of classifying any of these as a small-colony

TABLE 3

Further classification of progenies from crosses which were not segregating for small-colony

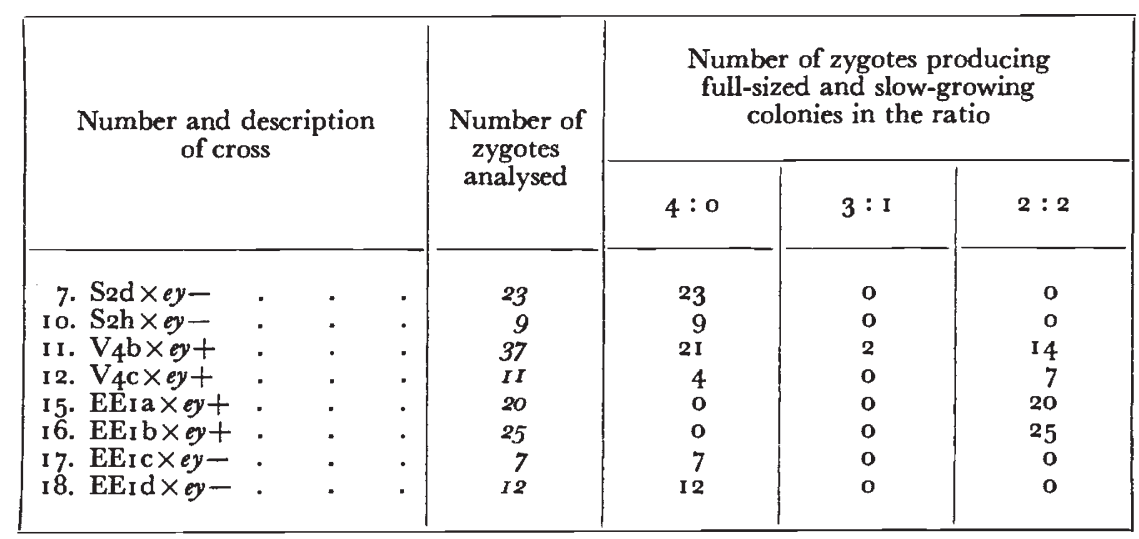

type. Nevertheless in certain of the progenies some variation in colony size was apparent. In these cases either four or occasionally only two colonies from the set of eight were noticeably smaller than their fellows, having only about three-quarters their diameter. It was therefore possible to amplify the classification of zygote progenies to include this character, which is referred to as slow-growth. In table 3 the progenies from those crosses not also segregating for smallcolony are classified in this way.

Since each of the crosses in table 3 had one parent with the eyespot and one without, each progeny showed $2: 2$ segregation for presence and absence of the eyespot. When this was confirmed by examination of cells under the microscope it was found that all slow-growing clones lacked eyespots, though not all eyeless clones were slow-growing. This is a precisely similar situation to that found with small-colony clones, and a similar explanation appeared probable. 
The recognition of the slow-growth character prompted a closer examination of growth-rate in the two eyeless strains which have been used throughout this work. Whenever a cross has involved an eyeless strain of minus mating-type, a subculture from the original mutant strain has been used. Colonies of this strain cannot be distinguished from wild-type colonies except by examination of individual cells under the microscope. The eyeless strain of plus mating-type used in cross no. 2 and subsequently, was selected from the progeny of cross no. I. By comparison with wild-type colonies grown under similar conditions it has now been established that this eyeless plus strain shows slow-growth, though this passed unnoticed at the time of its selection. Since the progeny of cross no. I was segregating for small-colony, the presence of the less spectacular character, slow-growth, escaped attention.

In planning crosses to explain the inheritance of slow-growth it was postulated that a gene (symbol $s g$ ) causes slow growth in presence of the eyeless gene but is suppressed by its wild-type allele. On the basis of this hypothesis the crosses dealt with in table 3 are explained as follows :-

Crosses 7 and 10 : Neither parent carried $s g$, so no slow-growing clones appeared in any zygote progenies. Both crosses may therefore be written:

$$
e y^{+} s g^{+} \times e y s g^{+}
$$

Crosses 11 and 12: The eyeless parent carried $s g$ but the other did not. In those progenies where $e y$ and $s g$ remained associated, slow-growing clones appeared; but in others $s g$ was recombined with its suppressor $e y^{+}$, and slow-growth was not expressed. Both crosses may be written :

$$
e y^{+} s g^{+} \times e y s g
$$

Crosses 15-1 8 : Zygote EE 1 was heterozygous for the $s g$ locus, so that clones (a) and (b) received $s g$ but clones (c) and (d) received the alternative allele $s g^{+}$. Clones (a) and (b) were crossed with eyeless plus carrying $s g$, so that all zygotes in these crosses (nos. 15 and 16 ) were homozygous for $s g$ and all progenies included slowgrowing clones. Crosses 15 and 16 may therefore be written :

$$
e y+s g \times e y s g
$$

Clones (c) and (d) were crossed with eyeless minus, so that all zygotes were homozygous for $s g^{+}$and no slow-growing clones were produced. Crosses 17 and 18 may be written :

$$
e y^{+} s g^{+} \times e y s g^{+}
$$

According to the hypothesis all zygotes from cross no. II (table 3) have the genotype $e y^{+} s g^{+} / e y s g$. In the case of zygotes which produced eight full-sized colonies, the clones with eyespots should have the genotype $e^{+} s g$. If these clones were crossed with eyeless minus (carrying $s g^{+}$) the progenies should include some recombinant clones of genotype ey $\mathrm{sg}$ which are slow-growing. Suitable clones of plus mating-type were selected from the progeny of cross no. I I and crossed with eyeless minus; the results are shown in table 4 (crosses 19 and 20 ).

Other zygotes from cross no. I I produced four full-sized colonies 
and four which were slow-growing. In these cases the full-sized colonies are postulated to have the genotype $\mathrm{ey}^{+} \mathrm{sg}^{+}$, and when crossed with eyeless minus they should not yield any slow-growing clones at all. Suitable clones of plus mating-type were selected and crossed with eyeless minus; the results are shown in table 4 (crosses $2 \mathrm{I}$ and 22).

All zygotes from cross no. 15 (table 3 ) are expected by hypothesis to have the genotype $e y^{+} s g / e y s g$, and all clones in their progenies should carry sg. Those with eyespots have full-sized colonies, but when crossed with eyeless minus they are expected to yield some recombinant, slow-growing clones of genotype ey sg. Crosses of this kind were made with the results shown in table 4 (crosses 23 and 24).

All of the results in table 4 are consistent with expectations. Slowgrowth is thus similar to small-colony in its relation to eyeless.

Progenies have been examined from 151 zygotes heterozygous at the $e y$ and $s g$ loci. They consist of 59 showing parental two-type,

TABLE 4

Results of crosses made to study the inheritance of slow-growth

\begin{tabular}{|c|c|c|c|c|c|}
\hline \multirow{2}{*}{\multicolumn{2}{|c|}{$\begin{array}{c}\text { Number and description } \\
\text { of cross }\end{array}$}} & \multirow{2}{*}{$\begin{array}{l}\text { Number of } \\
\text { zygotes } \\
\text { analysed }\end{array}$} & \multicolumn{3}{|c|}{$\begin{array}{l}\text { Number of zygotes producing } \\
\text { full-sized and slow-growing } \\
\text { colonies in the ratio }\end{array}$} \\
\hline & & & $4: 0$ & $3: 1$ & $2: 2$ \\
\hline $\begin{array}{l}\text { 19. } 35.5 \times e y- \\
\text { 20. } 35.7 \times e y- \\
21.35 .1 \times e y- \\
22.35 .2 \times e y- \\
23.35 .11 \times e y- \\
24.35 .12 \times y=\end{array}$ & $:$ & $\begin{array}{l}34 \\
22 \\
21 \\
13 \\
24 \\
24\end{array}$ & $\begin{array}{r}11 \\
6 \\
21 \\
13 \\
8 \\
10\end{array}$ & $\begin{array}{l}0 \\
1 \\
0 \\
0 \\
4 \\
2\end{array}$ & $\begin{array}{r}23 \\
15 \\
0 \\
0 \\
12 \\
12\end{array}$ \\
\hline
\end{tabular}

83 non-parental two-type and 9 four-type segregations. The two classes of two-type segregations are expected to be equally frequent if the loci are unlinked. There is here a significant deviation from a I : I ratio but it is in the opposite direction to that expected if the loci are linked. It is concluded that $e y$ and $s g$ are not linked, but some mechanism may exist whereby the progeny of certain crosses includes a high frequency of non-parental combinations.

The low frequency of four-type segregations $(5.96$ per cent.) indicates that both loci are close to their centromeres and therefore show a low frequency of second-division segregation.

\section{DISCUSSION}

Since the original mutant clone produced colonies indistinguishable from those of the wild-type except by microscopic examination of individual cells, it can be established that it had the genotype 
ey $s c^{+} s g^{+}-$. In cross no. I (table I) this strain was crossed with the wild-type strain of plus mating-type and the progeny included some clones showing the small-colony character and others showing slowgrowth (see p. 244). It follows that at least one parent of the cross carried the genes $s c$ and $s g$, and since it is known that the eyeless parent did not, it must be that the wild-type parent did. This parent therefore had the genotype $e y^{+} s c s g+$, which gives normal growth rate since $s c$ and $s g$ are suppressed by $e y^{+}$.

Cross no. 2 and similar crosses made subsequently also gave rise to small-colony clones though neither parent showed this character. This means that the wild-type strain of minus mating-type carried the sc gene. Some slow-growing clones are expected in the progeny of cross no. 2 since the eyeless parent was itself slow-growing. If the $s g$ gene were carried by the eyeless parent only, some recombinant clones of genotype ey $\mathrm{sc}^{+} \mathrm{sg}^{+}$with normal growth rate would be expected also, but no eyeless clones with normal growth rate have ever been recovered from crosses of this type. It seems highly probable therefore that the wild-type strain of minus mating-type carried the sg gene.

The hypothesis of a multiple mutation having occurred emerges from the observation that irradiation of a clone of genotype $e y+s c s g$ (the wild-type minus strain) has led to the recovery of a clone with the genotype ey $s c^{+} s g^{+}$(the eyeless mutant). If mutations at these three loci are independent of each other the possibility of simultaneous mutation at all three is remote but not unreal. Alternatively one might postulate some mutational relationship between the three loci. This intentionally vague suggestion takes into account the possibility that the loci show similar instability towards a particular mutagenic agent. It also allows the existence of some mechanism whereby mutation at one locus is accompanied by or increases the likelihood of mutation at the other two. There is ample evidence from work on other organisms that mutations at different loci are not always independent of each other, though no case is known to the author which is closely comparable to that described here.

Frankel (1950) lists several instances where simultaneous mutation of both alleles at a locus has been reported, and himself describes a chlorophyll-deficient type of wheat which differs from both parents by genes at three loci. These loci show independent inheritance, but since wheat is hexaploid it is not unlikely that the three loci are homologous. The evidence is compatible with simultaneous mutation of both alleles at all three loci. The appearance in the mutant plants of a large inverted chromosome duplication is regarded by Frankel as more than a coincidence, and he interprets the structural change as a possible "destabiliser" of heredity. The work of McClintock (I95I) on chromosome rearrangement in relation to mutability lends considerable support to this interpretation. It is not known if there is any structural change in the chromosomes of the eyeless mutant 
of $C$. reinhardi. The small size of the cells makes it doubtful whether the chromosomes could be studied in detail.

Mampell ( 1945 , I946) presents evidence for a substance in Drosophila which causes mutability at a number of different loci in different chromosomes. The majority of genes affected by this mutator produce a minute phenotype when mutated, and may therefore be related in some way other than their positions on the chromosomes. In $D$. pseudoobscura formation of the mutator depends on a recessive gene and the presence of a Y-chromosome, but when transferred to $D$. melanogaster the substance is self-reproducing. It is conceivable that the three mutations believed to have occurred in Chlamydomonas were produced, not by the direct action of ultra-violet radiation on the loci concerned, but by a secondary mutagen, itself the product of ultraviolet radiation, to which all three loci are susceptible. The physiological relationship which has been demonstrated between these three loci may be of significance in this respect.

Tedin and Hagberg (I952) describe the results of treating seeds of Lupinus luteus with X-rays. One of the plants grown from these seeds was shown to carry three recessive mutant genes which, when homozygous, severally cause reduced chlorophyll content, lack of basal branches, and sterility. None of the genes was known to be present in the original material, and there is strong evidence that all three are on the same chromosome. The plants have a chromosome number of $5^{2}$ so that while the chance of producing three independent mutations is itself very small, the chance that all three will be on the same chromosome is infinitesimal. It is concluded that the three mutations are not independent of each other and that linkage has made possible "some kind of chain reaction in one chromosome, or some other complicated phenomenon". It is unlikely that a similar event has occurred in Chlamydomonas since there is evidence that neither $s c$ nor $s g$ is linked to $e y$, though in the absence of data to the contrary the possibility that they are linked to each other cannot be ruled out.

\section{SUMMARY}

Following irradiation of wild-type cells of Chlamydomonas reinhardi a mutant clone lacking the eyespot has been isolated. Presence or absence of the eyespot behaves as a single gene difference. Crosses between this mutant (eyeless) and the wild-type have revealed the existence of two genes affecting colony size, which are suppressed by the wild-type allele at the eyeless locus. The characters concerned are called small-colony and slow-growth. The wild-type strains carry both these genes but also carry the suppressor, so the characters are not expressed. After mutation to the eyeless condition, the original mutant clone no longer carried the genes for reduced colony size but alternative alleles giving normal colonies. It is deduced that mutation at the eyeless locus was accompanied by mutation at the other two loci. 


\section{REFERENCES}

FRANKEL, O. H. 1951. A polymeric multiple gene change in hexaploid wheat. Heredity, 4, 103-1 16.

MAMPELL, X. 1945. Analysis of a mutator. Genetics, 30, 496-505.

MAMPELL, K. 1946. Genic and non-genic transmission of mutator activity. Genetics, 31, 589-597.

McClintock, B. 1951. Chromosome organization and genic expression. Cold Spring Harbor Symp., I6, $13-47$.

SMITH, G. M., AND REGNERY, D. C. 1950. Inheritance of sexuality in Chlamydomonas reinhardi. P.N.A.S., 36, 246-248.

TEDIN, O., AND HAGBERG, A. 1952. Studies on X-ray induced mutations in Lupinus luteus L. Hereditas, 38, 267-296.

Whitehouse, H. L. K. 1949. Multiple-allelomorph heterothallism in the fungi. New Phytol., 48, 21 2-244. 